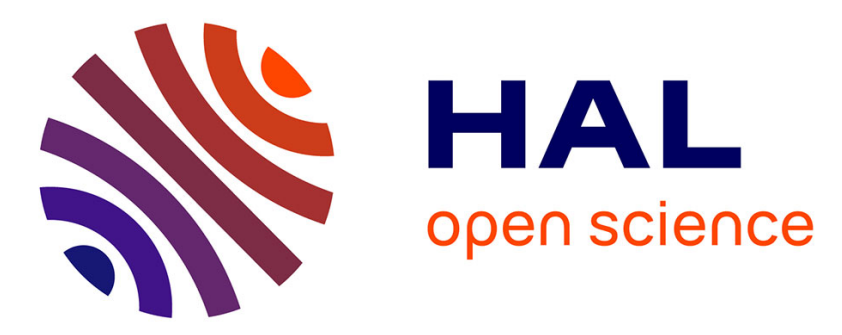

\title{
On the design of efficient Vehicular Applications
}

Yacine Khaled, Manabu Tsukada, José Santa Lozano, Thierry Ernst

\section{To cite this version:}

Yacine Khaled, Manabu Tsukada, José Santa Lozano, Thierry Ernst. On the design of efficient Vehicular Applications. IEEE Vehicular Technology Conference, Apr 2009, Barcelona, Spain. inria00355878

\section{HAL Id: inria-00355878 \\ https://hal.inria.fr/inria-00355878}

Submitted on 26 Jan 2009

HAL is a multi-disciplinary open access archive for the deposit and dissemination of scientific research documents, whether they are published or not. The documents may come from teaching and research institutions in France or abroad, or from public or private research centers.
L'archive ouverte pluridisciplinaire HAL, est destinée au dépôt et à la diffusion de documents scientifiques de niveau recherche, publiés ou non, émanant des établissements d'enseignement et de recherche français ou étrangers, des laboratoires publics ou privés. 


\title{
On the design of efficient Vehicular Applications
}

\author{
Yacine Khaled*, Manabu Tsukada*, José Santa ${ }^{\dagger}$, Thierry Ernst* \\ *INRIA Rocquencourt, IMARA Team \\ Domaine de Voluceau \\ BP 105, 78153 Le Chesnay Cedex, France \\ Email: (yacine.khaled|manabu.tsukada|thierry.ernst)@inria.fr \\ ${ }^{\dagger}$ Department of Information and Communications Engineering \\ Computer Science Faculty \\ University of Murcia \\ Campus de Espinardo, 30100 Murcia, Spain \\ Email: josesanta@um.es
}

\begin{abstract}
Vehicular communications attract the attention of many people in the networking research world. These networks present some special features, such as high mobility or specific topologies, which affect the performance of applications. In order to select the

appropriate technologies, more effort should be directed to identify the final necessities of the network. Few works identify possible applications of vehicular networks, but none of them link application requirements which networking technologies available in the vehicular field. In this paper, we fill this gap, and propose an analysis of application requirements and study how to deal with them using communication technologies for the physical and network level. This study contains key factors which must be taken into account, especially, at the designing stage of the vehicular network.
\end{abstract}

\section{INTRODUCTION}

Nowadays, communications become essential in the information society. People can connect to data networks anywhere, even in mobility conditions, by means of different devices and communication technologies. The vehicle is a place where users stay many time everyday. Hence, offering on-board networked applications is a challenge for the scientific community. In addition to safety applications (the most studied ones), other telematic services could improve traffic efficiency, environment preservation and comfort. However, vehicular networks needed to design such applications are characterized by special features, far away of from common fixed and, even, ad-hoc networks characteristics. New mobility patterns and topologies could affect the performance and feasibility of some applications.

In our opinion, the identification of telematic application requirements, and the study of what communication technology can fulfil them, are key factors which have to be considered at the design stage. This is the only way of assuring a good performance in vehicular application from early stages. Following this idea, the work presented in this paper analyses the main networking requirements of vehicular applications, and gives some guidelines about how to cover them with current wireless communication technologies and networking solutions. However, as starting point, it is interesting to study current trends in vehicular applications and communications.

In this paper, first, vehicular applications and services are presented in section [I] Then, the most common communication technologies in the vehicular field are introduced in section III In section IV, we start by analysing the usage of communication technologies, efficiently, to fill applications needs. Then, we introduce the networking technologies and analyse them in the same context. However, before these analysis, the most important application requirements are presented. Finally, some concluding remarks end the paper.

\section{VEHICULAR APPLICATIONS AND SERVICES}

According to the current literature, vehicular applications can be divided in two main families: safety and non-safety. Road safety applications are the more important concern in Intelligent Transport Systems (ITS). Many people die or result injured in traffic accidents every day. Vehicular communications can help us to anticipate the problem, extending the road visibility by disseminating safety information. Several applications for increasing the road safety can be enabled by vehicular networks, such as cooperative collision avoidance (either for intersection or highway), lateral collision warning, incident management, emergency video streaming, etc.

In addition to road safety applications, traffic information and monitoring systems are other important issue in vehicular networks, whose aim is focused on improving the traffic flow and road usage. Inside this group of services it is worth quoting platooning, cooperative notification systems, vehicle tracking, lane change assistance, road network monitoring, traffic jam prevention, or weather information.

Finally, comfort applications, whose main goal is offering novel services on-board, have been recently proposed, apart from common multimedia capabilities. These applications improve driver and passengers conditions by means of Internet access, distributed games, instant messaging, tourist and leisure information, movie announces, or parking booking.

In order to analyse this wide range of vehicular applications, we target three of the most important applications for each of these families: safety, traffic management and monitoring, and, finally, comfort services.

a) Safety applications:

Cooperative collision warning. These applications enhance the driver conditions by monitoring the distance between vehicles and, depending on the implementation, warn the driver or automatically break when the distance decreases under a certain threshold. These systems also take into account the post-collision situation, when vehicles near the accident must be warned.

Incident management. The aim of this system is to successfully manage current accidents on the road. First, by detecting road problems (e.g. obtaining location and nature of accident) via satellite positioning or other sensors. The next point is to manage vehicle flows during and after the accident, through vehicular communications.

Emergency video streaming. It deals with video information forwarding in emergency contexts. Some vehicles are equipped 
with video cameras and have enough storage capabilities to buffer and forward the images. This service can be provided over V2V (vehicle to vehicle) communications.

b) Traffic management and monitoring systems:

Platooning. Such systems allow vehicles to travel closely and safely in an efficient way. This leads to a reduction in the space used by vehicles on a highway. In consequence, more vehicles can use the highway without causing traffic congestions.

Vehicle tracking. These services allow car manufacturers, logistic companies and other trusted parties, to remotely monitor vehicle statistics.

Notification services. These applications consists of providing travel information to users through an Internet access. After the subscription, users can be notified when information is available. As application examples, we can quote weather and traffic forecasting.

c) Comfort applications:

Parking place management. This service allows drivers to discover a free parking place and booking it. Additionally, a vehicle could park itself without the need of driver assistance.

Distributed games and/or talks. This kind of entertainment applications comprise the management of activities between a limited group of vehicles in a distributed fashion, and via V2V. For instance, we can quote card games, sharing draws or instantaneous talks.

Peer to peer applications. Using these services it is possible to share information between vehicles, without contacting any application server. Applications such as instantaneous messaging, file transfer and voice over IP are the most considered.

\section{Communication technologies}

In order to deploy telematic vehicular applications, an underlying communication platform is necessary. Current Level $1 / 2$ wireless technologies considered in vehicular communications can be divided in two groups: those which can work establishing a direct vehicle to vehicle (V2V) link, and those which need the support of infrastructure's equipment. Communication with the infrastructure can use uplink (V2I) and/or downlink (I2V) channels. We can summarise the wireless technologies used in the vehicle field as the next ones (see Fig. 1):

Bluetooth. Although Bluetooth was specially created for short range communications with peripherals, it is possible to create small vehicular networks using it. However, coverage limitations (in the order of tens of meters) limit the wide applicability of Bluetooth in vehicular communications.

WLAN and DSRC. Wireless Local Area Networks (WLAN) comprises a set of standards inside the 802.11 group. Although their main usage lies on a managed communication mode, they also allow an ad-hoc mode, useful in V2V networks. $802.11 \mathrm{a} / \mathrm{b} / \mathrm{g}$ specifications offer tens of Mbps over distances around 100 metres. Dedicated Short Range Communications (DSRC) comprise similar technologies, but more adapted to the vehicle domain. The new 802.11p is being used as background in the DSRC research, covering the transmission of critical information in timely-aware vehicular applications.

Cellular networks. New UMTS (Universal Mobile Telecommunications System) networks offers data connections of several Mbps, and their wide deployment and mobility advantages are important features for vehicular communications. However, coverage and delay are still the main drawbacks of cellular networks.

WiMAX. The Worldwide Interoperability for Microwave Access (WiMAX) comprises a communication technology which try to fill the gap between $3 \mathrm{G}$ and WLAN. The $802.16 \mathrm{e}$ standard has been specially designed for mobile users connected to a base station, and represents a potential technology for vehicular communications. Its tens of Mbps, mobility up to $100 \mathrm{Km} / \mathrm{h}$, and $10 \mathrm{Km}$ of coverage to the base station, make this solution suitable for urban scenarios.

RDS and TMC. The Radio Data System (RDS) was developed to carry digital data using the common FM radio band. TMC (Traffic Message Channel) usually considers RDS to send traffic information messages, which can be used by navigation devices to calculate alternative routes, for instance.

Satellite. Satellite communications can be used in I2V data transmissions, similar to RDS/TMC. However, the areas covered by satellites are even higher than in FM. Uplink communications are also possible, but the hardware is too expensive and bulky to be mounted in vehicles.

\section{COVERING NETWORKING NEEDS EFFICIENTLY}

\section{A. Vehicular applications requirements}

Prior to select a communication technology or a network architecture for a new project, it is necessary to study main requirements of applications from a networking point of view. Next we include some of the most important ones.

Location awareness. Reliable and scalable communication capabilities are required in order to ensure the exchange of information among vehicles. Critical safety services such as alert cooperative collision warning and incident management need a high accurate localization, as well as some comfort applications such as parking booking. Other services, however, require a low accurate localization, like peer to peer applications and vehicle tracking. Additional positioning features of communication technologies can be very useful when GPS is not available.

Geocast capability. Geocast considers the transmission of messages within a geographical region [1], [2]. The complexity of defining this region can be as high as the set of vehicles behind or in front the subject one. Other times this constraint is relaxed, and defining this region as the vehicles inside a geographic area, or near a designated spot (such as a smog area), is enough. Geocast is considered efficient when the information could be forwarded in both sparse and dense geographical areas, while efficiently leveraging the available bandwidth.

Penetration rate dependency. This parameter may have important consequences in the operation of some applications [3], especially the critical and safety ones. Although a low penetration rate is obviously a problem in safety applications, such as collision avoidance, an excess of equipped vehicles also arises transmission troubles. Note that penetration rate has a direct bearing on the wireless bandwidth used. The higher the penetration rate, the higher the wireless bandwidth necessary to allow vehicles to communicate.

Time awareness One of the most important criterions for measuring the quality of the network is communication delay. Although most applications have time constraints, those related with road safety are critical. Due to this, a challenge in vehicular networks is providing real-time capabilities. In order to enable the driver to react quickly, information must reach vehicles with a low delay. However, this requirement is difficult to ensure in mobile networks, and even more in vehicular networks.

Permanent access. In infrastructure-based networks, operators do not offer the same service over the entire terrestrial surface. Urban environments do not present coverage problems, but in rural locations the deployment is sometimes poor. A vehicle equipped with a VANET system, however, is always able to emit 
messages, because the vehicle itself is part of the network "infrastructure". At the application level, some services such as file transfer or video conference, need a permanent communication channel.

Mobility. In 802.11 transmissions, distance between sender and receiver is an important factor for the transmission rate obtained [4]. In infrastructure-based technologies, handoffs between base stations lowers the network performance. Poor latency and throughput results are obtained if the mobile terminal is moving at locations far away from the UMTS Node B without performing a handoff [5]. Interference with other radio equipments in the case of VANET should also be taken into account, due to the wide usage of the $2.4 \mathrm{GHz}$ frequency band [6]. Other external factors, like the existence of other vehicles or buildings, are considered in realistic mobility patterns for VANET solutions [7].

\section{B. Requirements fulfilled by communication technologies}

Regarding wider range communication technologies, such as satellite, RDS, cellular and WiMAX, some common aspects can be found considering the application requirements they can fulfill. All of them can avoid penetration rate problems present in short range contexts (e.g. WLAN, Bluetooth and DSRC). This is solved by the necessary infrastructure which provides the access to the network, which also enables a quasi permanent access to the network with high degrees of mobility. However, the amount of users simultaneously connected to the network is limited by the available bandwidth. RDS, on the contrary, does not present this problem because only permits traffic in broadcast, as it is also noticeable in most of satellite deployments. Regarding geocasting, all wide-range technologies can simulate this feature by means of base station broadcasting. However, the performance of this method is limited by the size of coverage areas, which sometimes can be too large, as occurs in the extreme case of satellite communications. An overlay network like the one presented in [8] can solve this problem. Location functionalities are possible in cellular and WiMAX networks, following a detection mechanism at the base station. Cellular networks take advantage of this method in most urban environments to provide a good approximation to the user position. Finally, it is important to treat the delay in these wide-range technologies. Real time services could be considered by using cellular and, overall, WiMAX. However, in the case of safety services, the infrastructure available in the region of interest has to be evaluated.

WLAN takes advantage of the infrastructure and ad hoc operation modes, to improve the penetration rate dependency by combining these two communication modes. In infrastructure mode, the access point can contribute to improve the location accuracy and provide geocast capabilities, as it is noticeable in the GeoNet Project [9]. DSRC, and more specifically IEEE 802.11p, allows the implementation of critical applications such as safety services, considering real-time constraints. This is carried out by using a specific emergency channel and some priority levels for the traffic. Even if Bluetooth technology is limited by its range and connection time, it can be used when vehicles are very close, to maintain the connection to the network and alleviate the penetration rate problem.

\section{Networking (L3) solutions for vehicles}

Main network (level-three) technologies treated in standardisation bodies for vehicular communications are considered to study how they can treat several application requirements.

MANET and VANET. Mobile Ad hoc Networks (MANET) are suitable for wireless routing applications with dynamic

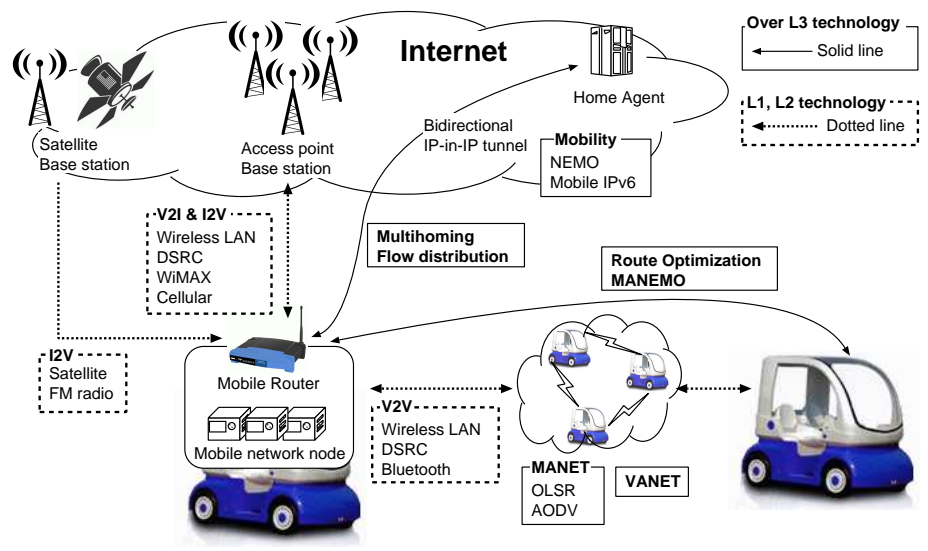

Fig. 1. Overview of the technologies

topologies. This type of communication does not require any infrastructure. In order to route messages in such a network, each node is invited to participate in the message forwarding. Vehicular Ad hoc NETworks (VANET), a particular case of MANET, are characterized by a strong mobility of the nodes, a high dynamic topology, a significant loss rate, and a very short duration of communication. In these networks, the node location is never stable, either locally or globally, and routing messages is a great challenge. Many works have been done to design ad hoc routing algorithms to deal with the node's mobility, periodically updating routing tables by means of proactive algorithms (e.g. OLSR and FASTOLSR [10], [11]); discovering routes by using reactive approaches (.e.g AODV [12]); using geographical information to improve routing (e.g. GAMER, LBM [1], GPSR [13]); detecting stable structures, or clusters [14], [15], [1]; using the node's movement for transporting messages [16], [17], [18], [19]; following a broadcast approach for messages forwarding [20], [21], [22], [23], etc. Note that some of these works have been introduced in standardisation bodies (like ETSI [24]), vehicular consortiums (C2C-CC [25]), and European projects (GeoNet).

As explained in section IV-A V2V communications are highly dependent on the penetration rate. It is difficult to ensure a good communication under low and high load. To reduce this dependence, some solutions have been proposed. For example, in high penetration conditions, Geocast approaches can use the directed flooding approach, where the closer vehicle to the destination one retransmit the message (e.g. LBM [1]). Under low load conditions, some solutions have been proposed, where the messages progress towards the destination by means of node's movements [26], [19], [17], [27]. These solutions enable also to take into account real-time constraints, by optimizing communications. In applications requiring a permanent access, VANET can be seen as an important asset in places where infrastructure-based networks are not available.

NEMO. The NEMO Basic Support [28] functionalities involve a router on the Internet to allow mobile computers to maintain a global connectivity. In the ITS field, the basic scheme is represented in Fig. 11 A Mobile Router (MR) located in the vehicle acts as a gateway for the Mobile Network of the vehicle, and manages mobility on behalf of its Mobile Network Nodes (MNN). The MR and a fixed router in the Internet, called Home Agent (HA), establish a bi-directional tunnel which is used to transmit the packets between the MNN and their Correspondent Nodes $(\mathrm{CN})$.

Although the location information is still lacking in NEMO (or IPv6 in general), many solutions and project deal with the geographical location. First, NEMO can be implemented over 
geographical routing [29], [30], [9], to improve routing and offer geocast communication. Other approach consist of extending IPv6 with geographical information [31], [32]

Multihoming. MRs can be shipped with multiple network interfaces such as IEEE802.11 a/b/g, WiMAX, GPRS/UMTS, etc. When a MR maintains these interfaces simultaneously up and has multiple paths to the Internet, it is said to be multihomed. In mobile environments, MRs often suffer from scarce bandwidth, frequent link failures and limited coverage. Multihoming brings the benefits of alleviating these issues [33].

The maintenance of multiple network interfaces deals with permanent access requirement, because several communication technologies can be used at the same time. For WiMAX or cellular can be used when a high bandwidth connection through WLAN is not available, for example. Also, multihoming can help to choose the appropriate interface according to the penetration rate situation.

Flow distribution. To transfer data through multiple interfaces, a policy based flow distribution mechanism should be used. The traffic can be distributed by multiple paths considering the source and destination addresses, source and destination ports or traffic type. In NEMO basic support, traffic from the Internet to the mobile network is distributed by the HA, while the distribution in the opposite direction is carried out at the MR. An efficient traffic distribution technique can increase the bandwidth considerably, and decrease the communication delay.

Route Optimization. NEMO is one of the main level-three technologies of vehicle communication, however, some issues related to Route Optimization still remain unsolved in NEMO Basic Support, while they have already been covered in Mobile IPv6 [34]. In NEMO, all the packets to and from MNNs must be encapsulated and sent by means of an IPs tunnel between the MR and the HA. Thus, all these packets between MNNs and CNs must go through the HA. This arises several performance issues. In IETF, route optimization issues of NEMO are addressed in [35]. Requirements of route optimization in various scenarios are described for vehicular networks in [36], and for aeronautic environments in [37]. Solving route optimization problems we can improve the network performance, mainly reducing transmission delay, which is of great interest in time awareness applications.

MANEMO. Both MANET and NEMO are designed independently as layer-three technologies. NEMO is designed to provide global connectivity, and MANET to provide direct routing in localised networks. MANEMO (MANET and NEMO) comprises the usage of both concepts together, which could bring benefits for route optimization. Since direct routes are available in MANET local networks, MANET can provide direct paths between vehicles, as Fig. 1 shows. This mechanism can be used to treat permanent access requirements of some applications.

P2P overlay network over cellular networks. The usefulness of cellular networks in an architecture which allows communications between vehicles and with the infrastructure is presented in [8]. The network architecture uses a P2P approach over the cellular network basis to enable vehicles to receive and send data packets. This architecture covers a location aware capabilities of traffic information services, and offers an overlay approximation for geocasting.

The relation among applications, requirements and technologies are summarized in the Table I. This table is divided into two parts. The first part evaluates the degree of presence, in the applications described above, of each requirement. For instance, we can notice that location awareness and geocast capability could be considered as key requirements in safety applications. On the other hand, they are less important in other types of applications.

The second part of Table I illustrates the relation between requirements and technologies. For example, we can notice that VANET communication could improve the location and time awareness and geocast capability, and NEMO could enhance the mobility and ensure a permanent Internet access. We can notice also that VANET communication and NEMO are very complementary in order to fill these requirements.

\section{CONCLUSION}

Many vehicular applications and services have been proposed at the beginning of this paper. However, in order to allow this services to efficiently work, we should take into account the networking requirements of such applications, and know how to fulfil them with current technologies. This is the aim of the analysis presented in current paper.

An initial overview of applications and services classifies them into three main families: safety, traffic management and monitoring, and comport. Then, for each family, we describe the three reference applications which best represent it in the current literature. All of these have specific requirements far away from traditional services in fixed networks. To meet these demands, a number of wireless communication technologies at level one/two are appropriate for the vehicle domain. The paper analyses what application requirements can be fulfilled with them and, because many necessities cannot be covered by level $1 / 2$ technologies, main level 3 network solutions considered inside IETF (and some other ITS organisations) have been presented. Since no specific technology can satisfy all the requirements, our opinion is that future vehicles will combine several of them in order to enable the deployment of different applications inside the vehicle.

Engineers should take into account both application requirements and available technologies for designing new ITS solutions. Our contribution is focused on helping engineers in the design of application/services for testbeds or commercial products.

\section{ACKNOWLEDGEMENTS}

The authors would like to thank the Spanish Ministerio the Educación y Ciencia for sponsoring the research activities under the grant AP2005-1437, in frames of the FPU program.

\section{REFERENCES}

[1] C. Maihöfer. A survey of geocast routing protocols. IEEE Communications Surveys and Tutorials, 6, 2nd quarter 2004.

[2] Harshvardhan P.J. Distributed robust geocast: A multicast protocol for intervehicle communication. Master's thesis, Dep. of Electrical and Computer Engineering, NCSU, 2006.

[3] S. Breitenberger, B. Grüber, M. Neuherz, and R. Kates. Traffic information potential and necessary penetration rates. Traffic engineering \& control, 45(11):396-401, July 2004.

[4] Y. Khaled, B Ducourthial, and M. Shawky. IEEE 802.11 performances for inter-vehicle communication networks. In Proc. of th 61st IEEE Semianual Vehicular Technology conference VTC, Stockholm, Sweden, 2005-Spring.

[5] A. Alexiou, C. Bouras, and V. Igglesis. Performance evaluation of tcp over umts transport channels. In International Symposium on Communications Interworking, Ottawa, Canada, November/December 2004.

[6] C. Wewetzer, M. Caliskan, K. Meier, and A. Luebke. Experimental evaluation of umts and wireless lan for inter-vehicle communication. In International Conference ITS Telecommunications, Sophia Antipolis, France, June 2007.

[7] V. Naumov, R. Baumann, and T Gross. An evaluation of inter-vehicle ad hoc networks based on realistic vehicular traces. In ACM International Symposium on Mobile Ad Hoc Networking and Computing, Florence, Italia, May 2006. 
TABLE I

RELATION AMONG APPLICATIONS, REQUiREMENTS AND TECHNOLOGIES

\begin{tabular}{|c|c|c|c|c|c|c|c|}
\hline \multicolumn{2}{|c|}{ Requirements } & $\begin{array}{c}\text { Location } \\
\text { awareness }\end{array}$ & $\begin{array}{c}\text { Geocast } \\
\text { capability }\end{array}$ & $\begin{array}{c}\text { Penatration } \\
\text { rate dependency }\end{array}$ & $\begin{array}{c}\text { Time } \\
\text { awareness }\end{array}$ & $\begin{array}{c}\text { Permanent } \\
\text { Internet access }\end{array}$ & Mobility \\
\hline \multicolumn{8}{|c|}{ Applications } \\
\hline Safety & $\begin{array}{l}\text { Cooperative collision warning } \\
\text { Incident management } \\
\text { Emergency video streaming }\end{array}$ & $\begin{array}{l}\text { *** } \\
\text { *** } \\
\text { *** }\end{array}$ & $\begin{array}{l}\text { *** } \\
\text { *** } \\
\text { *** }\end{array}$ & $\begin{array}{l}\text { *** } \\
\text { *** } \\
\text { *** }\end{array}$ & $\begin{array}{l}\text { *** } \\
\text { *** } \\
\text { *** }\end{array}$ & $\begin{array}{l}* \\
* \\
*\end{array}$ & $\begin{array}{l}\text { *** } \\
* * \\
* *\end{array}$ \\
\hline Traffic & $\begin{array}{c}\text { Platooning } \\
\text { Vehicles tracking } \\
\text { Notification Services }\end{array}$ & $\begin{array}{l}* * \\
* \\
* \\
*\end{array}$ & ** & $\begin{array}{l}* * \\
* \\
*\end{array}$ & $\begin{array}{l}\text { *** } \\
\text { * } \\
*\end{array}$ & $\begin{array}{l}* \text { * } \\
\text { ** } \\
*\end{array}$ & $\begin{array}{l}* * \\
* \\
*\end{array}$ \\
\hline Comfort & $\begin{array}{l}\text { Parking place management } \\
\text { Distributed game } \\
\text { Peer to peer }\end{array}$ & $\begin{array}{l}* * \\
* \\
*\end{array}$ & $\begin{array}{l}\text { *** } \\
\text { * }\end{array}$ & $\begin{array}{l}\text { * } \\
* \\
* \\
*\end{array}$ & $\begin{array}{l}* \\
* \\
*\end{array}$ & $\begin{array}{l}* * \\
* \\
* *\end{array}$ & $\begin{array}{l}\text { *** } \\
\text { ** } \\
*\end{array}$ \\
\hline \multicolumn{8}{|c|}{$\begin{array}{l}\text { none }=\text { Requirement not present } \\
*=\text { Requirement present } \\
* * \text { = Key requirement }\end{array}$} \\
\hline \multicolumn{8}{|c|}{ Technologies } \\
\hline $\begin{array}{l}\text { L1, } \\
\text { L2 }\end{array}$ & $\begin{array}{l}\text { PAN (ex. Bluetooth, UWD ...) } \\
\text { LAN (ex. WLAN, DSRC ...) } \\
\text { MAN (ex. WiMAX, Cellular ...) } \\
\text { RDS/TMC, Satellite }\end{array}$ & $\begin{array}{l}\text { Possible } \\
\text { Possible } \\
\text { Possible } \\
\quad \times\end{array}$ & $\begin{array}{l}\text { Help } \\
\text { Help }\end{array}$ & $\begin{array}{l}\text { Sensitive } \\
\text { Sensitive } \\
\text { Sensitive }\end{array}$ & Possible & $\begin{array}{l}\text { Help } \\
\text { Help } \\
\text { Help } \\
\text { Help }\end{array}$ & $\begin{array}{l}\text { } \\
\bigcirc \\
\bigcirc\end{array}$ \\
\hline L3 & $\begin{array}{c}\text { VANET } \\
\text { NEMO } \\
\text { Multihoming } \\
\text { Route optimization } \\
\text { MANEMO }\end{array}$ & 0 & 0 & Sensitive & $\bigcirc$ & $\begin{array}{c}\text { Help } \\
\bigcirc \\
\bigcirc\end{array}$ & $\bigcirc$ \\
\hline
\end{tabular}

\begin{tabular}{|c|c|c|}
\hline ) & & gy fills the requirement (The $r$ \\
\hline & - & gy cannot fill the requirement \\
\hline ossible & $=$ & $\begin{array}{l}\text { The technology can be used to fill the requirement with other technologies } \\
\text { (The requirement cannot be fill by only the technologies) }\end{array}$ \\
\hline Sensitive & $=$ & $\begin{array}{l}\text { Penetration rate dependency sensitive } \\
\text { (Note: no technology can increase penetration rate, } \\
\text { people equips it because it is cheep or useful, for example.) }\end{array}$ \\
\hline elp & $=$ & $\begin{array}{l}\text { The technology help the requirement } \\
\text { (The requirement cannot be filled by only the technology) }\end{array}$ \\
\hline
\end{tabular}

[8] J. Santa and A.F. Gomez-Skarmeta. Architecture and evaluation of a unified V2V and V2I communication system based on cellular networks. Elsevier Computer Communications, 31(12):2850-2861, July 2008.

[9] Geonet project.

[10] T. Clausen, P. Jacquet, A. Laouiti, P. Muhlethaler, A. Qayyum, and L. Viennot. Optimized link state routing protocol. In Procs. of IEEE INMIC, Pakistan, 2001.

[11] M. Benzaid, P. Minet, and K. Al Agha. Integrating fast mobility in the OLSR routing protocol. In IEEE MWCN, Stockholm, 2002.

[12] I.D. Chakeres and M.E. Belding-Royer. AODV routing protocol implementation design. In Procs. of WWAN, Tokyo, Japan, 2004.

[13] M. Mauve, J. Widmer, and H. Hartenstein. A survey on position-based routing in mobile ad hoc networks. IEEE Network Magazine, November/December 2001.

[14] M. Jiang, J. Li, and Y.C. Tay. Cluster based routing protocol (CBRP). Technical report, IETF, 2001. Internet draft.

[15] T.D.C. Little and A. Agarwal. An information propagation scheme for VANETs. In 8th International IEEE Conference on Intelligent Transportation Systems, Vienna, Austria, 2005.

[16] A. Vahdat and D. Becker. Epidemic routing for partially connected ad hoc networks. Technical Report CS-200006, Computer Science Dpt, Duke University, 2000.

[17] Q. Li and D. Rus. Sending messages to mobile users in disconnected adhoc wireless networks. In 6th annual international conference on Mobile computing and networking (MOBICOM), 2000.

[18] J. Davis, A. Fagg, and B. Levine. Wearable computers as packet transport mechanisms in highly-partitioned ad-hoc networks. In procs. of IEEE ISWC, Washington, DC, USA, 2001.

[19] J. Zhao and G. Cao. VADD: Vehicle-assisted data delivery in vehicular ad hoc networks. In Procs. of (INFOCOM), Barcelona, Spain, 2006.

[20] H. Alshaer and E. Horlait. An optimized adaptive broadcast scheme for inter-vehicle communication. In IEEE VTC, Stockholm, Sweden, 2005.

[21] G. Korkmaz, E. Ekici, F. Ozguner, and U. Ozguner. Urban multi-hop broadcast protocol for inter-vehicle communication systems. In the 1 st ACM international workshop on Vehicular ad hoc networks, Philadelphia, PA, USA, 2004

[22] M. Sun, W. Feng, T. Lai, K. Yamada, H. Okada, and K. Fujimura. Gps-based message broadcasting for inter-vehicle communication. In International Conference on Parallel Processing, Washington, DC, USA, 2000.
[23] S. Biswas, R. Tatchikou, and F. Dion. Vehicle-to-vehicle wireless communication protocols for enhancing highway traffic safety. IEEE Communications Magazine, 44(1):74-82, January 2006.

[24] ETSI: http://www.etsi.org.

[25] C2C-CC: http://www.car-to-car.org.

[26] T. Kosch. Technical concept and prerequisites of car-to-car communication Technical report, BMW Group Research and Technology, 2002.

[27] I. Chatzigiannakis, E. Nikoletseas, and P. Spirakis. An efficient communication strategy for ad-hoc mobile networks. In Procs. of DISC, London, UK, 2001

[28] V. Devarapalli, R. Wakikawa, A. Petrescu, and P. Thubert. Network Mobility (NEMO) Basic Support Protocol, January 2005. IETF RFC3963.

[29] R. Baldessari, A. Festag, A. Matos, J. Santos, and R. Aguiar. Flexible connectivity management in vehicular communication networks. In Proc. of the WIT, Hamburg, Germany, 2006.

[30] R. Baldessari, A. Festag, W. Zhang, and L. Le. A manet-centric solution for the application of nemo in vanet using geographic routing. In Proc. of th Weedev, Austria, 2008.

[31] L. Chen, J. Steenstra, and taylor K-S. Geolocation-based addressing method for ipv6 addresses. Patent, Qualcomm Incorporated, January 2008

[32] J. Vare, J. Syrjarinne, and K-S Virtanen. Geographical positioning extension for IPv6. In Proc. of the ICN, Guadeloupe, 2004.

[33] T. Ernst, N. Montavont, R. Wakikawa, C. Ng, and K. Kuladinithi. Motivations and Scenarios for Using Multiple Interfaces and Global Addresses, July 2007. IETF, draft-ietf-monami6-multihoming-motivation-scenario-02.

[34] D. Johnson, C. Perkins, and J. Arkko. Mobility Support in IPv6, June 2004 IETF RFC 3775.

[35] C. Ng, P. Thubert, M. Watari, and F. Zhao. Network Mobility Route Optimization Problem Statement, July 2007. IETF RFC4888.

[36] R. Baldessari, A. Festag, and M. Lenardi. C2C-C Consortium Requirements for NEMO Route Optimization, July 2007. IETF, draft-baldessari-c2cccnemo-req-01.

[37] W. Eddy, W. Ivancic, and T. Davis. NEMO Route Optimization Requirements for Operational Use in Aeronautics and Space Exploration Mobile Networks, December 2007. IETF, draft-ietf-mext-aero-reqs-00. 